\title{
Erratum to: The More the Merrier?
}

\section{Entropy and Statistics of Asexual Reproduction in Freshwater Planarians}

\author{
Sofia Quinodoz • Michael A. Thomas • Jörn Dunkel • \\ Eva-Maria Schötz
}

Published online: 11 October 2012

(C) Springer Science+Business Media New York 2012

\section{Erratum to: J Stat Phys (2011) 142:1324-1336 \\ DOI 10.1007/s10955-011-0157-3}

The original area data was calculated using the wrong conversion factor. The y-axis labels of Fig. 5A and 5B and the area values reported in Sects. 3.2 and 3.5 should be multiplied by a factor 4 to obtain the correct areas. The remaining results and the conclusions in the original article are not affected.

The online version of the original article can be found under doi:10.1007/s10955-011-0157-3.

S. Quinodoz · M.A. Thomas · E.-M. Schötz ( $\varangle)$

170 Carl Icahn Laboratory, Lewis-Sigler Institute, Princeton University, Princeton, NJ 08544, USA

e-mail: eschoetz@princeton.edu

S. Quinodoz

e-mail: quinodoz@princeton.edu

M.A. Thomas

e-mail: matthree@princeton.edu

J. Dunkel

Department of Applied Mathematics and Theoretical Physics, Centre for Mathematical Sciences,

University of Cambridge, Wilberforce Road, Cambridge CB3 0WA, UK

e-mail: j.dunkel@damtp.cam.ac.uk 